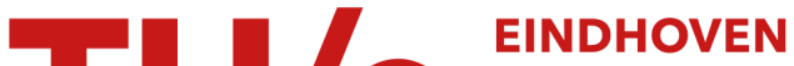 UNIVERSITY OF TECHNOLOGY
}

\section{Generating potentially nilpotent full sign patterns}

Citation for published version (APA):

Kim, I. J., Olesky, D. D., Shader, B. L., Driessche, van den, P., Holst, van der, H., \& Vander Meulen, K. N. (2009). Generating potentially nilpotent full sign patterns. Electronic Journal of Linear Algebra, 18, 162-175.

Document status and date:

Published: 01/01/2009

\section{Document Version:}

Publisher's PDF, also known as Version of Record (includes final page, issue and volume numbers)

\section{Please check the document version of this publication:}

- A submitted manuscript is the version of the article upon submission and before peer-review. There can be important differences between the submitted version and the official published version of record. People interested in the research are advised to contact the author for the final version of the publication, or visit the $\mathrm{DOI}$ to the publisher's website.

- The final author version and the galley proof are versions of the publication after peer review.

- The final published version features the final layout of the paper including the volume, issue and page numbers.

Link to publication

\section{General rights}

Copyright and moral rights for the publications made accessible in the public portal are retained by the authors and/or other copyright owners and it is a condition of accessing publications that users recognise and abide by the legal requirements associated with these rights.

- Users may download and print one copy of any publication from the public portal for the purpose of private study or research.

- You may not further distribute the material or use it for any profit-making activity or commercial gain

- You may freely distribute the URL identifying the publication in the public portal.

If the publication is distributed under the terms of Article $25 \mathrm{fa}$ of the Dutch Copyright Act, indicated by the "Taverne" license above, please follow below link for the End User Agreement:

www.tue.nl/taverne

Take down policy

If you believe that this document breaches copyright please contact us at:

openaccess@tue.nl

providing details and we will investigate your claim. 


\title{
GENERATING POTENTIALLY NILPOTENT FULL SIGN PATTERNS*
}

\author{
I.-J. KIM ${ }^{\dagger}$, D.D. OLESKY ${ }^{\ddagger}$, B.L. SHADER ${ }^{\S}$, P. VAN DEN DRIESSCHE ${ }^{\Uparrow}$, \\ H. VAN DER HOLST" ${ }^{\prime}$, AND K.N. VANDER MEULEN**
}

\begin{abstract}
A sign pattern is a matrix with entries in $\{+,-, 0\}$. A full sign pattern has no zero entries. The refined inertia of a matrix pattern is defined and techniques are developed for constructing potentially nilpotent full sign patterns. Such patterns are spectrally arbitrary. These techniques can also be used to construct potentially nilpotent sign patterns that are not full, as well as potentially stable sign patterns.
\end{abstract}

Key words. Potentially nilpotent, Spectrally arbitrary, Inertia, Potentially stable, Sign pattern.

AMS subject classifications. 15A18, 05C50.

1. Introduction. An $m$-by- $n$ matrix $\mathcal{A}=\left[\alpha_{i j}\right]$ with entries $\alpha_{i j} \in\{+,-, 0\}$ is called a sign pattern (matrix), and defines an associated sign pattern class of real matrices $Q(\mathcal{A})=\left\{A=\left[a_{i j}\right] \in \mathbb{R}^{m \times n}: \operatorname{sign} a_{i j}=\alpha_{i j} \forall i, j\right\}$. A matrix $A \in Q(\mathcal{A})$ is a realization of pattern $\mathcal{A}$. If no entry of $\mathcal{A}$ is zero, then $\mathcal{A}$ is a full sign pattern. If sign pattern $\mathcal{B}=\left[\beta_{i j}\right]$ has $\beta_{i j}=\alpha_{i j}$ whenever $\alpha_{i j} \neq 0$, then $\mathcal{B}$ is a superpattern of $\mathcal{A}$. Given a real matrix $A=\left[a_{i j}\right]$, its associated sign pattern, denoted by $\mathcal{S}(A)$, has $(i, j)$-entry equal to sign $a_{i j}$ for all $i, j$.

Associated with an $n$-by- $n$ sign pattern $\mathcal{A}$ is a signed directed graph with vertices $1, \ldots, n$ and a positive (negative) arc from $i$ to $j$ if and only if $\alpha_{i j}>0 \quad\left(\alpha_{i j}<0\right)$. For $q \geq 2$, if $i_{1}, \ldots, i_{q}$ are distinct and $\alpha_{i_{1}, i_{2}} \cdots \alpha_{i_{q-1}, i_{q}} \alpha_{i_{q}, i_{1}}>0 \quad(<0)$, then the signed directed graph has a positive (negative) $q$-cycle on vertices $i_{1}, \ldots, i_{q}$. If $\alpha_{i i}>$ $0\left(\alpha_{i i}<0\right)$, then the signed directed graph has a positive (negative) 1-cycle or loop at vertex $i$.

A sign pattern $\mathcal{A}$ is potentially nilpotent $(\mathrm{PN})$ if there exists a nilpotent matrix $A \in Q(\mathcal{A})$, i.e., $A^{t}=0$ for some positive integer $t$. If $t$ is the smallest integer such

*Received by the editors September 16, 2008. Accepted for publication March 5, 2009. Handling Editor: Michael J. Tsatsomeros.

$\dagger$ Dept. of Math. and Stat., Minnesota State University, Mankato, MN 56001, USA.

${ }^{\ddagger}$ Dept. of Comp. Sci., U. of Victoria, P.O. Box 3055, Victoria, BC, Canada V8W 3P6.

$\S$ Dept. of Math., University of Wyoming, Laramie, WY 82071, USA.

IDept. of Math. and Stat., U. of Victoria, P.O. Box 3060, Victoria, BC, Canada V8W 3R4.

$\|$ Dept. of Math. and Comp. Sci., Eindhoven U. of Tech., P.O. Box 513, 5600 MB Eindhoven, NL.

${ }^{* *}$ Dept. of Math., Redeemer University College, Ancaster, ON, Canada L9K 1J4. 
that $A^{t}=0$, then $t$ is the nilpotent index of $A$. $\mathcal{A}$ allows nilpotence of index $t$ if there is some matrix $A \in Q(\mathcal{A})$ with nilpotent index $t$. In this paper, we focus on full sign patterns that are potentially nilpotent. To put these in context, we introduce a few more definitions.

If a real $n$-by- $n$ matrix $A$ has $n_{+}, n_{-}$, and $n_{0}$ eigenvalues having positive, negative, and zero real parts, respectively, with $n_{+}+n_{-}+n_{0}=n$, then the ordered triple of nonnegative integers $\left(n_{+}, n_{-}, n_{0}\right)$ is the inertia of $A$, denoted by $i(A)$. The inertia of a sign pattern $\mathcal{A}$ is $\{i(A): A \in Q(\mathcal{A})\}$. Letting $\sigma(A)$ denote the spectrum of a matrix $A$, the spectrum of a sign pattern $\mathcal{A}$ is $\{\sigma(A): A \in Q(\mathcal{A})\}$. If $A$ has inertia $(0, n, 0)$, then $A$ is a stable matrix, and a pattern $\mathcal{A}$ is potentially stable if $\mathcal{A}$ allows inertia $(0, n, 0)$, i.e., there exists a stable matrix $A \in Q(\mathcal{A})$. If $\mathcal{A}$ allows all possible inertias, then $\mathcal{A}$ is an inertially arbitrary (sign) pattern ( $\mathcal{A}$ is an IAP), and if $\mathcal{A}$ allows all possible spectra for a real matrix, then $\mathcal{A}$ is a spectrally arbitrary (sign) pattern ( $\mathcal{A}$ is a SAP); see [8] where these definitions were introduced. The Nilpotent-Jacobian method was developed in [8] to show that a pattern $\mathcal{A}$ is a SAP by first finding a nilpotent matrix $A \in Q(\mathcal{A})$ and then verifying that a certain Jacobian matrix is nonsingular so that the Implicit Function Theorem can be used. For a precise statement of the NilpotentJacobian method, see [1, Lemma 2.1]. In [8] it was also observed that if $\mathcal{A}$ is proved to be a SAP by the Nilpotent-Jacobian method, then every superpattern of $\mathcal{A}$ is also a SAP. Thus much of the literature on SAPs has focused on sparse spectrally arbitrary patterns, although the first published $n$-by- $n$ spectrally arbitrary sign patterns were full sign patterns (see [15]).

If $D$ is an $n$-by- $n$ diagonal matrix with each diagonal entry +1 or -1 , then $A \mapsto D A D^{-1}$ is a signature similarity transformation, which we denote by a vector of the signs of the diagonal entries of $D$. If $P$ is an $n$-by- $n$ permutation matrix, then $A \mapsto P A P^{T}$ is a permutation similarity transformation. We use, for example, the notation (23) to refer to the permutation $P$ that interchanges rows two and three. The properties of being PN, an IAP and a SAP are invariant under negation, transposition, signature similarity transformations and permutation similarity transformations. Patterns $\mathcal{A}$ and $\mathcal{B}$ are equivalent if $\mathcal{A}$ can be obtained from $\mathcal{B}$ by any combinations of these four operations.

A sign pattern $\mathcal{A}$ is $\mathrm{PN}^{+}$if $\mathcal{A}$ is potentially nilpotent and the signed directed graph of $\mathcal{A}$ has at least one negative loop, one positive loop, and one negative 2-cycle. The following result, which is contained in [4], gives two necessary conditions for a sign pattern to be an IAP, and motivates the above definition of the class $\mathrm{PN}^{+}$; see also [7, Lemma 3.2].

THEOREM 1.1. If a sign pattern $\mathcal{A}=\left[\alpha_{i j}\right]$ is inertially arbitrary, then it has at least one positive and one negative entry on the main diagonal and at least one product $\alpha_{j k} \alpha_{k j}$ negative with $j \neq k$. 
If a sign pattern is spectrally arbitrary then it is PN. The converse is not true, but the following result of Pereira shows that the condition of being potentially nilpotent is a sufficient condition for a full sign pattern to be spectrally arbitrary.

Theorem 1.2. [17, Theorem 2.1] Any potentially nilpotent full sign pattern is spectrally arbitrary.

Both Pereira's theorem and the Nilpotent-Jacobian method emphasize the importance of finding nilpotent matrices and potentially nilpotent sign patterns in the study of spectrally arbitrary patterns. However, determining nilpotent matrices and PN sign patterns is not an easy task.

Our main aim is to construct potentially nilpotent full sign patterns so that, by Theorem 1.2, we can identify full sign patterns that are spectrally arbitrary. In Section 3, we give a construction that is also useful for other applications. For example, given a (not necessarily full) nilpotent matrix, our method can be used to generate a PN sign pattern (not necessarily full) of higher order. Similarly, the construction can be used to determine higher order potentially stable patterns from a given stable matrix.

2. Full sign patterns of orders $\mathbf{2}, \mathbf{3}$ and $\mathbf{4}$. For sign patterns of order 2, it is well known (see, for example, [8]) that up to equivalence, the only (full) sign pattern that is potentially nilpotent is

$$
\mathcal{T}_{2}=\left[\begin{array}{cc}
+ & + \\
- & -
\end{array}\right]
$$

and this pattern is also $\mathrm{PN}^{+}$, an IAP and a SAP. Thus these properties are equivalent for $n=2$.

For sign patterns of order 3, it was demonstrated in [1] and [4] that, up to equivalence, if a sign pattern is spectrally arbitrary, then it is a superpattern of one of the following four patterns:

$$
\begin{gathered}
\mathcal{D}_{3,3}=\left[\begin{array}{ccc}
- & + & 0 \\
- & 0 & + \\
- & 0 & +
\end{array}\right] \mathcal{T}_{3}=\left[\begin{array}{ccc}
- & + & 0 \\
- & 0 & + \\
0 & - & +
\end{array}\right] \\
\mathcal{U}_{3}=\left[\begin{array}{lll}
- & + & 0 \\
- & + & + \\
0 & + & -
\end{array}\right] \quad \mathcal{V}_{3}=\left[\begin{array}{lll}
- & 0 & + \\
- & 0 & + \\
- & + & +
\end{array}\right]
\end{gathered}
$$

Since each of these patterns can be proved to be spectrally arbitrary by using the Nilpotent-Jacobian method [8], it follows that every superpattern, and in particular 
every full superpattern, of each of these patterns is also spectrally arbitrary. However, not every full 3-by-3 pattern satisfying the necessary conditions in Theorem 1.1 is such a superpattern. This is demonstrated by the pattern

$$
\mathcal{R}=\left[\begin{array}{lll}
- & + & + \\
- & + & - \\
- & - & +
\end{array}\right],
$$

which is equivalent to the 3 -by-3 pattern given in [13, Theorem 5.1] that requires a positive eigenvalue, and hence is neither potentially nilpotent nor spectrally arbitrary.

TheOREM 2.1. Up to equivalence, $\mathcal{R}$ is the only full pattern of order 3 that satisfies the necessary conditions of Theorem 1.1 and is not spectrally arbitrary.

Proof. Suppose $\mathcal{A}=\left[\alpha_{i j}\right]$ is a full sign pattern that satisfies the necessary conditions and suppose $\mathcal{A}$ is not spectrally arbitrary. We can assume that up to permutation equivalence, $\alpha_{11}<0$, and $\alpha_{33}>0$. We consider the following three cases.

1. Suppose $\alpha_{12} \alpha_{21}<0$. Then, without loss of generality,

$$
\mathcal{A}=\left[\begin{array}{ccc}
- & + & * \\
- & * & * \\
* & * & +
\end{array}\right]
$$

where $* \in\{+,-\}$. Up to the signature similarity transformation $(+,+,-)$, it can be assumed that $\alpha_{32}<0$. If $\alpha_{13}<0$, then $\mathcal{A}$ is equivalent to a superpattern of $\mathcal{D}_{3,3}$, via transposition and the signature similarity transformation $(+,-,+)$. Thus $\alpha_{13}>0$. If $\alpha_{23}>0$, then $\mathcal{A}$ is a superpattern of $\mathcal{T}_{3}$; thus $\alpha_{23}<0$. If $\alpha_{31}>0$, then $\mathcal{A}$ is equivalent to a superpattern of $\mathcal{D}_{3,3}$ via the signature similarity transformation $(+,+,-)$. Thus $\alpha_{31}<0$. If $\alpha_{22}<0$ then $\mathcal{A}$ is equivalent to a superpattern of $\mathcal{U}_{3}$, via the permutation similarity transformation (23) and the signature similarity transformation $(+,+,-)$. Therefore $\alpha_{22}>0$, and $\mathcal{A}$ is equivalent to $\mathcal{R}$.

2. Suppose $\alpha_{23} \alpha_{32}<0$. Then $\mathcal{A}$ is equivalent to the sign pattern considered in Case 1 via negation and the permutation similarity transformation (13).

3. Suppose $\alpha_{13} \alpha_{31}<0$. Then, without loss of generality,

$$
\mathcal{A}=\left[\begin{array}{ccc}
- & * & + \\
* & * & * \\
- & * & +
\end{array}\right] .
$$

If $\alpha_{22}>0$, then via the permutation similarity transformation (23) a sign pattern in the form (2.1) is obtained. If $\alpha_{22}<0$, then via the permutation similarity transformation (23) followed by (12) and negation, a sign pattern in the form (2.1) is obtained. Thus $\mathcal{A}$ is equivalent to $\mathcal{R}$. 
A bordering technique is described in Section 3 that will enable us to easily construct most full spectrally arbitrary sign patterns of order 3 .

For sign patterns of order 4, using a computer search, we determined that every full sign pattern that satisfies the two necessary conditions of Theorem 1.1 is a SAP. In particular, we determined that every full sign pattern of order 4 that satisfies the two necessary conditions is a superpattern of one of the following four sign patterns:

$$
\begin{array}{rlr}
\mathcal{C}_{1} & =\left[\begin{array}{cccc}
0 & + & 0 & 0 \\
0 & - & + & + \\
+ & 0 & 0 & + \\
+ & - & 0 & +
\end{array}\right], & \mathcal{C}_{2}=\left[\begin{array}{cccc}
- & + & 0 & 0 \\
- & 0 & + & + \\
0 & 0 & + & + \\
- & 0 & 0 & 0
\end{array}\right], \\
\mathcal{C}_{3} & =\left[\begin{array}{cccc}
+ & - & 0 & 0 \\
+ & 0 & - & 0 \\
+ & 0 & 0 & - \\
0 & 0 & + & -
\end{array}\right], & \mathcal{C}_{4}=\left[\begin{array}{cccc}
- & + & 0 & 0 \\
- & 0 & + & 0 \\
0 & 0 & + & + \\
0 & - & + & 0
\end{array}\right] .
\end{array}
$$

These four sign patterns (and all their superpatterns) were shown to be spectrally arbitrary in the following referenced papers: $\operatorname{sign}$ pattern $\mathcal{C}_{1}$ is the first sign pattern on page 272 in [6, Appendix B], $\mathcal{C}_{2}$ is the first sign pattern in [6, Appendix $\left.\mathrm{A}\right], \mathcal{C}_{4}$ is the third sign pattern on page 271 in [6, Appendix $\mathrm{A}]$, and $\mathcal{C}_{3}$ is from [3]. To test these sign patterns, we first created a complete list of nonequivalent full sign patterns of order 4 that satisfy the two necessary conditions. Then we checked that each sign pattern in the list is equivalent to a superpattern of one of $\mathcal{C}_{i}$ for $i=1, \ldots, 4$.

3. Constructing potentially nilpotent full sign patterns. We now present a method for generating full potentially nilpotent sign patterns of higher order by bordering known potentially nilpotent sign patterns. We first introduce a new definition. The refined inertia $\operatorname{ri}(A)$ of a real $n$-by- $n$ matrix $A$ is the ordered 4 -tuple $\left(n_{+}, n_{-}, n_{z}, 2 n_{p}\right)$ such that $n_{+}$(resp. $n_{-}$) is the number of eigenvalues with positive (resp. negative) real part, and $n_{z}$ (resp. $2 n_{p}$ ) is the number of zero eigenvalues (resp. pure imaginary eigenvalues) of $A$. A pattern $\mathcal{A}$ allows refined inertia $\left(n_{+}, n_{-}, n_{z}, 2 n_{p}\right)$ if there exists $A \in Q(\mathcal{A})$ with $\operatorname{ri}(A)=\left(n_{+}, n_{-}, n_{z}, 2 n_{p}\right)$.

Theorem 3.1. Let $A$ be an n-by-n matrix, $U$ be an n-by-k matrix, $X$ be a $k$-by-n matrix and $K$ be a k-by-k matrix. If $B$ is the $(n+k)-b y-(n+k)$ matrix

$$
B=\left[\begin{array}{c|c}
A-U X & U \\
\hline X A-X U X-K X & X U+K
\end{array}\right],
$$

then $\sigma(B)=\sigma(A) \cup \sigma(K)$. In particular, $r i(B)=\operatorname{ri}(A)+\operatorname{ri}(K)$. 
Proof. Setting

$$
L=\left[\begin{array}{l|l}
I_{n} & O \\
\hline X & I_{k}
\end{array}\right] \quad \text { gives } \quad L\left[\begin{array}{l|l}
A & U \\
\hline O & K
\end{array}\right] L^{-1}=B
$$

Hence $B$ is similar to the reducible matrix $\left[\begin{array}{l|l}A & U \\ \hline O & K\end{array}\right]$.

In using the above theorem, if $\operatorname{ri}(K)=\left(\ell_{1}, \ell_{2}, m, 2 j\right)$, then it is often convenient to take

$$
K=a_{1} I_{\ell_{1}} \oplus\left(-a_{2}\right) I_{\ell_{2}} \oplus O_{m} \oplus\left[\begin{array}{cc}
0 & b_{1} \\
-b_{1} & 0
\end{array}\right] \oplus \cdots \oplus\left[\begin{array}{cc}
0 & b_{j} \\
-b_{j} & 0
\end{array}\right],
$$

where $a_{1}, a_{2}, b_{1}, \ldots, b_{j}$ are positive integers. (Note that if $m=0$, then the zero block is vacuous; similarly if $\ell_{1}=0$ or $\ell_{2}=0$, then an identity block is vacuous; and if $j=0$, then the 2 -by-2 blocks giving pure imaginary eigenvalues are vacuous.)

COROLlary 3.2. If the matrix $A$ in Theorem 3.1 is nilpotent of index $t$ and $K=O_{k}$, then $B$ given by (3.1) is nilpotent of index $t$ or $t+1$.

Proof. The fact that $B$ is nilpotent follows directly from Theorem 3.1, since $r i(A)=(0,0, n, 0), r i(K)=(0,0, k, 0)$ and $r i(B)=r i(A)+r i(K)$. Moreover,

$$
B^{t}=L\left[\begin{array}{c|c}
A^{t} & A^{t-1} U \\
\hline O & O
\end{array}\right] L^{-1} .
$$

Given that $A$ is nilpotent of index $t$, it follows that if $A^{t-1} U=O$, then $B$ is nilpotent of index $t$; whereas if $A^{t-1} U \neq O$, then $B$ is nilpotent of index $t+1$.

TheOREM 3.3. Let $\mathcal{A}$ be an $n$-by-n sign pattern with $A \in Q(\mathcal{A})$, and $\mathcal{U}$ be an $n$-by- $k$ sign pattern with $U \in Q(\mathcal{U})$. Suppose $X$ is a $k$-by-n real matrix with no zero entries in the products $X A$ and $X U$. Then the $(n+k)$-by- $(n+k)$ sign pattern

$$
\mathcal{B}=\left[\begin{array}{c|c}
\mathcal{A} & \mathcal{U} \\
\hline \mathcal{S}(X A) & \mathcal{S}(X U)
\end{array}\right]
$$

allows refined inertia $\operatorname{ri}(A)+\left(\ell_{1}, \ell_{2}, m, 2 j\right)$ for any nonnegative vector $\left(\ell_{1}, \ell_{2}, m, 2 j\right)$ with $\ell_{1}+\ell_{2}+m+2 j=k$.

Proof. Let $K$ be as in (3.2). Then, by Theorem 3.1, the matrix $B$ in (3.1) has refined inertia $r i(B)=\operatorname{ri}(A)+\left(\ell_{1}, \ell_{2}, m, 2 j\right)$. If the entries of $U$ are sufficiently small and the entries of $K$ are sufficiently smaller than those of $X U$, then $\mathcal{S}(B)=\mathcal{B}$. $\square$

Corollary 3.4. If $\mathcal{A}$ and $\mathcal{U}$ in Theorem 3.3 are full sign patterns, then $\mathcal{B}$ in $(3.4)$ is a full sign pattern. Moreover, if $A \in Q(\mathcal{A})$ is nilpotent and $K$ in $(3.2)$ is $O_{k}$, then $\mathcal{B}$ is a full potentially nilpotent sign pattern. 
To illustrate the above construction, we now give some examples.

ExAmple 3.5. Matrix

$$
A=\left[\begin{array}{rr}
1 & 1 \\
-1 & -1
\end{array}\right] \in Q\left(\mathcal{T}_{2}\right)
$$

is nilpotent of index 2. Take $X=\left[\begin{array}{ll}1 & 0\end{array}\right], U=\left[\begin{array}{ll}\frac{1}{2} & \frac{1}{2}\end{array}\right]^{T}$, and $K=0$. Then, by Corollary 3.4, the 3-by-3 full sign pattern

$$
\mathcal{B}=\left[\begin{array}{cc|c}
+ & + & + \\
- & - & + \\
\hline+ & + & +
\end{array}\right]
$$

is potentially nilpotent. Hence, Theorem 1.2 implies that $\mathcal{B}$ is spectrally arbitrary. In fact, $\mathcal{B}$ is equivalent to a superpattern of $\mathcal{U}_{3}$. Moreover, the sign pattern $\mathcal{B}$ allows nilpotence of index 3 but not index 2 since $\left(B^{2}\right)_{33} \neq 0$ for every $B \in Q(\mathcal{B})$.

Example 3.6. Take $A, X, K$ as in Example 3.5, but $U=\left[\frac{1}{2},-\frac{1}{2}\right]^{T}$ so that $A U=O$. The matrix from (3.1) is

$$
B=\left[\begin{array}{rrr}
\frac{1}{2} & 1 & \frac{1}{2} \\
-\frac{1}{2} & -1 & -\frac{1}{2} \\
\frac{1}{2} & 1 & \frac{1}{2}
\end{array}\right] \quad \text { and } \quad \mathcal{S}(B)=\left[\begin{array}{rrr}
+ & + & + \\
- & - & - \\
+ & + & +
\end{array}\right]
$$

Since $B$ is nilpotent of index $2, \mathcal{S}(B)$ allows nilpotence of index 2 . Here $B=$ $[1,-1,1]^{T}\left[\frac{1}{2}, 1, \frac{1}{2}\right]$ has rank 1 , illustrating a result in $[9$, Corollary 3.7$]$. Note that $\mathcal{S}(B)$ is equivalent to a superpattern of $\mathcal{V}_{3}$.

We now demonstrate that our construction as in Example 3.6 can be viewed as an extension of the method for sign patterns that allow nilpotence of index 2 given in [9, Theorem 3.9], and for sign patterns that allow nilpotence of index 3 given in [10, Construction Method 3].

THEOREM 3.7. If a sign pattern $\mathcal{A}=\left[\begin{array}{ll}\mathcal{A}_{1} & \mathcal{A}_{2} \\ \mathcal{A}_{3} & \mathcal{A}_{4}\end{array}\right]$ allows nilpotence of index $t$ where $\mathcal{A}_{1}$ and $\mathcal{A}_{4}$ are square, then

$$
\hat{\mathcal{A}}=\left[\begin{array}{ccc}
\mathcal{A}_{1} & \mathcal{A}_{2} & \mathcal{A}_{2} \\
\mathcal{A}_{3} & \mathcal{A}_{4} & \mathcal{A}_{4} \\
\mathcal{A}_{3} & \mathcal{A}_{4} & \mathcal{A}_{4}
\end{array}\right] \quad \text { and } \quad \breve{\mathcal{A}}=\left[\begin{array}{rrr}
\mathcal{A}_{1} & \mathcal{A}_{2} & \mathcal{A}_{2} \\
\mathcal{A}_{3} & \mathcal{A}_{4} & \mathcal{A}_{4} \\
-\mathcal{A}_{3} & -\mathcal{A}_{4} & -\mathcal{A}_{4}
\end{array}\right]
$$

allow nilpotence of index $t$.

Proof. Suppose $A \in Q(\mathcal{A})$ is nilpotent of index $t$. Take $U=\left[\begin{array}{ll}A_{2} & A_{4}\end{array}\right]^{T}, \quad X=$ $\left[O, \frac{1}{2} I\right]$ and $K=O$ where the dimensions are compatible, and apply Corollary 3.2. 
Since $A$ is nilpotent of index $t$ and $U$ is composed of columns of $A$, it follows that $\hat{\mathcal{A}}$ allows nilpotence of index $t$. In particular, refering to (3.3), we need only demonstrate that $A^{t-1} U=O$. If $A$ is partitioned into block columns as $A=[W U]$, then it follows that $A^{t-1} U=O$ since

$$
O=A^{t}=A^{t-1}\left[\begin{array}{ll}
W & U
\end{array}\right]=\left[\begin{array}{ll}
A^{t-1} W & A^{t-1} U
\end{array}\right]
$$

Replacing $X$ by $-X$ in the above shows that $\breve{\mathcal{A}}$ also allows nilpotence of index $t$.

The constructions in Theorem 3.7 can be repeated to build sign patterns of higher order of the form

$$
\left[\begin{array}{cccc}
\mathcal{A}_{1} & \mathcal{A}_{2} & \cdots & \mathcal{A}_{2} \\
\mathcal{A}_{3} & \mathcal{A}_{4} & \cdots & \mathcal{A}_{4} \\
\vdots & \vdots & \ddots & \vdots \\
\mathcal{A}_{3} & \mathcal{A}_{4} & \cdots & \mathcal{A}_{4}
\end{array}\right]
$$

in which each row of block submatrices has a \pm sign (subject to $\mathcal{A}$ being potentially nilpotent). Note that this can give $p$-striped sign patterns (i.e., $n$-by- $n$ sign patterns with $p$ columns having all entries positive and $n-p$ columns having all entries negative with $1 \leq p \leq n-1$ ), the first family of sign patterns shown to be spectrally arbitrary [15].

The next result follows by taking $k=n, X=I_{n}$ (or $X=-I_{n}$ ), $K=O_{n}$ and $U$ sufficiently small in Theorem 3.1; see also Corollary 3.4.

THEOREM 3.8. If $\mathcal{A}$ is an $n$-by-n potentially nilpotent sign pattern, then

$$
\mathcal{B}_{1}=\left[\begin{array}{cc}
\mathcal{A} & \mathcal{U} \\
\mathcal{A} & \mathcal{U}
\end{array}\right] \quad \text { and } \quad \mathcal{B}_{2}=\left[\begin{array}{rr}
\mathcal{A} & \mathcal{U} \\
-\mathcal{A} & -\mathcal{U}
\end{array}\right]
$$

are $2 n$-by- $2 n$ potentially nilpotent sign patterns for any $n$-by- $n$ sign pattern $\mathcal{U}$. Moreover, if $\mathcal{A}$ and $\mathcal{U}$ are full patterns, then both $\mathcal{B}_{1}$ and $\mathcal{B}_{2}$ are full $2 n$-by-2n potentially nilpotent sign patterns.

$$
\begin{aligned}
& \text { EXAMPLE 3.9. Let } \mathcal{A}=\left[\begin{array}{ccc}
+ & + & + \\
- & - & - \\
- & - & -
\end{array}\right] \text { and } \mathcal{U}=\left[\begin{array}{ccc}
+ & + & + \\
+ & + & + \\
+ & + & +
\end{array}\right] \text {. If } \\
& A=\left[\begin{array}{rrr}
2 & 2 & 2 \\
-1 & -1 & -1 \\
-1 & -1 & -1
\end{array}\right], \quad U=\left[\begin{array}{lll}
1 & 1 & 1 \\
1 & 1 & 1 \\
1 & 1 & 1
\end{array}\right], \quad X=I_{3} \quad \text { and } \quad K=O_{3}
\end{aligned}
$$


then the matrix of the form in (3.1)

$$
\left[\begin{array}{ll}
(A-U) & U \\
(A-U) & U
\end{array}\right]=\left[\begin{array}{rrr|rrr}
1 & 1 & 1 & 1 & 1 & 1 \\
-2 & -2 & -2 & 1 & 1 & 1 \\
-2 & -2 & -2 & 1 & 1 & 1 \\
\hline 1 & 1 & 1 & 1 & 1 & 1 \\
-2 & -2 & -2 & 1 & 1 & 1 \\
-2 & -2 & -2 & 1 & 1 & 1
\end{array}\right]
$$

is nilpotent. Hence, the 6-by-6 full sign pattern

$$
\mathcal{B}_{1}=\left[\begin{array}{ll}
\mathcal{A} & \mathcal{U} \\
\mathcal{A} & \mathcal{U}
\end{array}\right]=\left[\begin{array}{ccc|ccc}
+ & + & + & + & + & + \\
- & - & - & + & + & + \\
- & - & - & + & + & + \\
\hline+ & + & + & + & + & + \\
- & - & - & + & + & + \\
- & - & - & + & + & +
\end{array}\right]
$$

is potentially nilpotent and so by Theorem 1.2, is spectrally arbitrary.

The methods above can also be applied to generate non-full nilpotent matrices and potentially nilpotent sign patterns from those of lower order. We illustrate this in the following example. The construction could be useful in implementing the Nilpotent Jacobian method to show that a non-full sign pattern is spectrally arbitrary.

EXAMPLE 3.10. Let $C_{i}$ for $i=1, \ldots, 4$ be a nilpotent realization of $\mathcal{C}_{i}$, a 4 by-4 SAP as defined in Section 2, and $U$ be any 4-by-4 matrix. Then by (3.1) with $A=C_{i}, X=I_{4}$ and $K=O_{4}$, the 8-by-8 matrix given by

$$
\left[\begin{array}{ll}
\left(C_{i}-U\right) & U \\
\left(C_{i}-U\right) & U
\end{array}\right]
$$

is nilpotent, and specifies a (not necessarily full) potentially nilpotent sign pattern of order 8 .

Returning to sign patterns of order 3 , there are, up to equivalence, exactly two full potentially nilpotent sign patterns that cannot be obtained by the bordering technique described in Theorem 3.1 with $K=O$. One of them is

$$
\mathcal{G}=\left[\begin{array}{l|l}
\mathcal{G}_{11} & \mathcal{G}_{12} \\
\hline \mathcal{G}_{21} & \mathcal{G}_{22}
\end{array}\right]=\left[\begin{array}{cc|c}
+ & + & + \\
- & - & + \\
\hline+ & - & -
\end{array}\right] .
$$

Any nilpotent realization $G_{11} \in \mathcal{G}_{11}$ has $G_{11}=\left[\begin{array}{rr}a & a \\ -a & -a\end{array}\right]$ for $a>0$. Thus for any realization $G_{21}\left(\right.$ resp. $\left.G_{12}\right)$ of $\mathcal{G}_{21}$ (resp. $\left.\mathcal{G}_{12}\right), G_{21}$ (resp. $G_{12}$ ) is not in the row (resp. 
column) space of $G_{11}$. Hence, the constructions described above cannot be used to prove that $\mathcal{G}$ is potentially nilpotent. However, $\mathcal{G}$ is equivalent to a superpattern of $\mathcal{T}_{3}$. Hence, the full sign pattern $\mathcal{G}$ is spectrally arbitrary. The other example is

$$
\mathcal{H}=\left[\begin{array}{ccc}
- & + & - \\
- & - & + \\
- & + & +
\end{array}\right]
$$

The full sign pattern $\mathcal{H}$ has no 2-by-2 potentially nilpotent principal submatrix, but is a spectrally arbitrary pattern since $\mathcal{H}$ is a superpattern of $\mathcal{D}_{3,3}$.

4. Refined inertia. A sign pattern $\mathcal{A}$ of order $n$ is a refined IAP (or an rIAP for short) if for every ordered 4-tuple $\left(n_{+}, n_{-}, n_{z}, 2 n_{p}\right)$, of nonnegative integers with $n_{+}+n_{-}+n_{z}+2 n_{p}=n$, there is some matrix $A \in Q(\mathcal{A})$ such that $A$ has $n_{+}$eigenvalues with positive real parts, $n_{-}$eigenvalues with negative real parts, $n_{z}$ zero eigenvalues and $2 n_{p}$ nonzero pure imaginary eigenvalues. The presence of nonzero pure imaginary eigenvalues is often of importance in the analysis of dynamical systems, because they can signal the onset of periodic solutions by Hopf bifurcation; see, for example [18, Appendix A.7].

The result of Theorem 3.1 can also be used to construct a matrix with a pair of pure imaginary eigenvalues, and thus identify other refined inertias for a pattern. In particular, we now show that the sign pattern $\mathcal{B}_{1}$ in Example 3.9 allows refined inertia $(0,0,4,2)$.

ExAmple 4.1. Let $A, U$ and $X$ be as in Example 3.9, but $K=\left[\begin{array}{rrr}0 & \frac{1}{2} & 0 \\ -\frac{1}{2} & 0 & 0 \\ 0 & 0 & 0\end{array}\right]$. Then the matrix

$$
B=\left[\begin{array}{rrr|rrr}
1 & 1 & 1 & 1 & 1 & 1 \\
-2 & -2 & -2 & 1 & 1 & 1 \\
-2 & -2 & -2 & 1 & 1 & 1 \\
\hline 1 & \frac{1}{2} & 1 & 1 & \frac{3}{2} & 1 \\
-\frac{3}{2} & -2 & -2 & \frac{1}{2} & 1 & 1 \\
-2 & -2 & -2 & 1 & 1 & 1
\end{array}\right] \in Q\left(\mathcal{B}_{1}\right)
$$

has eigenvalues $0,0,0,0$, and $\pm \frac{1}{2} i$.

By taking $A$ and $K$ to have nonzero pure imaginary eigenvalues, it is also possible to use the construction in Theorem 3.1 to identify matrices of even order $n \geq 4$ that have all nonzero pure imaginary eigenvalues. In a similar way, by taking $A$ and $K$ to be stable matrices, a stable matrix of higher order can be generated, thus giving a sign pattern that is potentially stable. In general, it is an open problem to 
characterize potentially stable sign patterns. Such sign patterns of orders $2,3,4$ with signed directed graphs that have only $r$-cycles with $r=1$ and $r=2$ (called tree sign patterns) are listed in [11], [14] and [16]. The following example uses a stable matrix of order 4 with signed directed graph having $r$-cycles with $r=1,2$ and $3[16$, Figure A2] to construct a potentially stable sign pattern of order 5 with these $r$-cycles.

ExAmPle 4.2. Take the stable matrix A given by

$$
A=\left[\begin{array}{rrrr}
0 & 1 & 0 & 1 \\
0 & 0 & 1 & 0 \\
-1 & 0 & 0 & 0 \\
-3 & 0 & 0 & -1
\end{array}\right], \quad X=\left[0,0,0, \frac{1}{2}\right], \quad U=[0,0,0,1]^{T}
$$

and $K=-\frac{1}{2}$. Then using Theorem 3.1, the matrix

$$
B=\left[\begin{array}{rrrrr}
0 & 1 & 0 & 1 & 0 \\
0 & 0 & 1 & 0 & 0 \\
-1 & 0 & 0 & 0 & 0 \\
-3 & 0 & 0 & -\frac{3}{2} & 1 \\
-\frac{3}{2} & 0 & 0 & -\frac{1}{2} & 0
\end{array}\right] \quad \text { with } \quad \mathcal{S}(B)=\left[\begin{array}{rrrrr}
0 & + & 0 & + & 0 \\
0 & 0 & + & 0 & 0 \\
- & 0 & 0 & 0 & 0 \\
- & 0 & 0 & - & + \\
- & 0 & 0 & - & 0
\end{array}\right]
$$

is stable, and specifies a potentially stable sign pattern of order 5 . By continuity, every superpattern (including full patterns) of $\mathcal{S}(B)$ is also potentially stable.

We conclude with some results that relate refined inertia to the other classes that we are considering. First note that if $\mathcal{A}$ is an $\operatorname{rIAP}$, then $\mathcal{A}$ is an IAP and $\mathrm{PN}^{+}$. If $\mathcal{A}$ is a sign pattern of order 2 or an irreducible pattern of order 3 , then the following are equivalent (see [1] and [4]): $\mathcal{A}$ is a $\mathrm{SAP}, \mathcal{A}$ is an IAP, $\mathcal{A}$ is $\mathrm{PN}^{+}, \mathcal{A}$ is an rIAP. We show by some examples that this equivalence does not hold in general for sign patterns of order 4 .

Example 4.3. Consider

$$
\mathcal{N}=\left[\begin{array}{cccc}
+ & + & 0 & 0 \\
0 & 0 & + & + \\
- & - & 0 & 0 \\
0 & 0 & - & -
\end{array}\right]
$$

From [4] we note that $\mathcal{N}$ is an IAP and $P N^{+}$but not a SAP. In fact, $\mathcal{N}$ does not allow a characteristic polynomial $x^{4}+r_{1} x^{3}+r_{2} x^{2}+r_{4}$ with $r_{4}>0$. Further, $\mathcal{N}$ is not an rIAP since $\mathcal{N}$ does not allow refined inertia $(0,0,0,4)$ as it does not allow a characteristic polynomial $x^{4}+c x^{2}+d$ with $c, d>0$. 
EXAmple 4.4. Consider

$$
\mathcal{N}_{2,1}=\left[\begin{array}{cccc}
+ & + & + & 0 \\
- & - & - & 0 \\
0 & 0 & 0 & + \\
- & - & 0 & 0
\end{array}\right] \quad \text { and } \quad \mathcal{N}_{2,2}=\left[\begin{array}{cccc}
+ & + & + & 0 \\
- & - & - & 0 \\
0 & 0 & 0 & - \\
- & - & 0 & 0
\end{array}\right]
$$

It was determined in [5] that $\mathcal{N}_{2,1}$, and $\mathcal{N}_{2,2}$ are inertially arbitrary and potentially nilpotent, but not spectrally arbitrary. We now show that these patterns are not in the class of rIAPs. Note that $\mathcal{M}_{4}$ from [y] is signature similar to $\mathcal{N}_{2,2}$.

Suppose $j \in\{1,2\}$ and $A \in Q\left(\mathcal{N}_{2, j}\right)$. By positive diagonal similarity, we may assume

$$
A=\left[\begin{array}{rrrr}
a & 1 & b & 0 \\
-c & -d & -1 & 0 \\
0 & 0 & 0 & v \\
-1 & -t & 0 & 0
\end{array}\right]
$$

with $a, b, c, d, t>0$ and $(-1)^{j+1} v>0$. Then

$$
p_{A}(x)=x^{4}+(d-a) x^{3}+(c-a d) x^{2}+v(b-t) x+v(b d+a t-c t b-1) .
$$

Suppose $r_{1}=0$ and $r_{3}=0$, where $r_{i}$ is the coefficient of $x^{4-i}$. Then $a=d$ and $b=t$ and

$$
p_{A}(x)=x^{4}+\left(c-d^{2}\right) x^{2}+(-v)\left(c b^{2}-2 b d+1\right) .
$$

Thus if $r_{2} \geq 0$ then $c=d^{2}+\epsilon$ for some $\epsilon \geq 0$. Hence $r_{4}=(-v)\left[d^{2}\left(b-\frac{1}{d}\right)^{2}+b^{2} \epsilon\right]$, so that $r_{4}=0$ or $\operatorname{sign}\left(r_{4}\right)=-\operatorname{sign}(v)$. It follows that $\mathcal{N}_{2,1}$ will not realize polynomials with $\operatorname{sign}\left(r_{1}, r_{2}, r_{3}, r_{4}\right) \in\{(0,0,0,+),(0,+, 0,+),(0,+, 0,0)\}$ and $\mathcal{N}_{2,2}$ will not realize polynomials with $\operatorname{sign}\left(r_{1}, r_{2}, r_{3}, r_{4}\right) \in\{(0,0,0,-),(0,+, 0,-),(0,+, 0,0)\}$. Since $\mathcal{N}_{2, j}$ cannot obtain sign $\left(r_{1}, r_{2}, r_{3}, r_{4}\right)=(0,+, 0,0), \mathcal{N}_{2, j}$ cannot realize polynomials of the form $x^{4}+r_{2} x^{2}$ with $r_{2}>0$. Thus $\mathcal{N}_{2, j}$ cannot realize the refined inertia $\left(n_{+}, n_{-}, n_{z}, n_{p}\right)=(0,0,2,2)$, and so $\mathcal{N}_{2, j}$ is not an rIAP.

The following diagram illustrates the relationship among the sign patterns discussed.

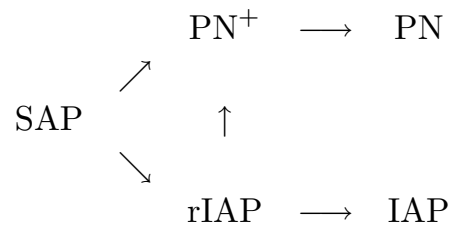

For a full sign pattern $\mathcal{A}$, it follows from Theorem 1.2 that the following statements are equivalent: $\mathcal{A}$ is a $\mathrm{SAP}, \mathcal{A}$ is $\mathrm{PN}^{+}, \mathcal{A}$ is $\mathrm{PN}$, and $\mathcal{A}$ is an rIAP. However we do not 
know whether these statements are equivalent to " $\mathcal{A}$ is an IAP" when $\mathcal{A}$ is a full sign pattern. Except possibly for the statement " $\mathcal{A}$ is an rIAP implies that $\mathcal{A}$ is a SAP", the converse of each implication in the diagram above is, in general, false. A family of sign patterns of odd order $n \geq 5$ that is inertially arbitrary but not potentially nilpotent is presented in [12].

5. Open questions. The above results lead to the following open questions.

(a) Is an inertially arbitrary full sign pattern necessarily PN, and hence a SAP?

(b) If a sign pattern is an rIAP, is it necessarily a SAP?

(c) Is any full $n$-by- $n, n \neq 3$, sign pattern that satisfies the two necessary conditions of Theorem 1.1 potentially nilpotent (and hence a SAP)? See Section 3 for some results for $n=2$ and 4 .

(d) Our construction can be used to show that certain superpatterns of direct sums of potentially nilpotent sign patterns allow nilpotence. Does every such superpattern allow nilpotence? For example, is every superpattern of $\mathcal{T}_{2} \oplus \mathcal{T}_{2}$ potentially nilpotent?

Acknowledgement. We wish to thank the American Institute of Mathematics where this research was started; see [2]. The research of DDO, PvdD, and KVM is partially supported by NSERC Discovery Grants. The work of IJK is partially supported by a Faculty Research Grant at MSU.

\section{REFERENCES}

[1] T. Britz, J.J. McDonald, D.D. Olesky, and P. van den Driessche. Minimal spectrally arbitrary patterns. SIAM Journal on Matrix Analysis and Applications, 26:257-271, 2004.

[2] R.A. Brualdi, L. Hogben, and B.L. Shader. AIM Workshop: Spectra of families of matrices described by graphs, digraphs and sign patterns; Final Report: Mathematical Results, March 30, 2007. Available at [http://aimath.org/pastworkshops/matrixspectrumrep.pdf].

[3] M.S. Cavers, I.-J. Kim, B.L. Shader, and K.N. Vander Meulen. On determining minimal spectrally arbitrary patterns. Electronic Journal of Linear Algebra, 13:240-248, 2005.

[4] M.S. Cavers and K.N. Vander Meulen. Spectrally and inertially arbitrary sign patterns. Linear Algebra and its Applications, 394:53-72, 2005.

[5] M.S. Cavers and K.N. Vander Meulen. Inertially arbitrary nonzero patterns of order 4. Electronic Journal of Linear Algebra, 16:30-43, 2007.

[6] L. Corpuz and J.J. McDonald. Spectrally arbitrary zero-nonzero patterns of order 4. Linear Algebra and its Applications, 55:249-273, 2007.

[7] L.M DeAlba, I.R. Hentzel, L. Hogben, J.J. McDonald, R. Mikkelson, O. Pryporova, B.L. Shader, and K.N. Vander Meulen. Spectrally arbitrary patterns: reducibility and the $2 n$ conjecture for $n=5$. Linear Algebra and its Applications, 423:262-276, 2007.

[8] J.H. Drew, C.R. Johnson, D.D. Olesky, and P. van den Driessche, Spectrally arbitrary patterns. Linear Algebra and its Applications, 308:121-137, 2000.

[9] C.A. Eschenbach and Z. Li. Potentially nilpotent sign pattern matrices. Linear Algebra and its Applications, 299:81-99, 1999.

[10] Y. Gao, Z. Li, and Y. Shao. Sign patterns allowing nilpotence of index 3. Linear Algebra and 
its Applications, 424:55-70, 2007.

[11] C.R. Johnson and T.A. Summers. The potentially stable tree sign patterns for dimensions less than 5. Linear Algebra and its Applications, 126:1-13, 1989.

[12] I.-J. Kim, D.D. Olesky, and P. van den Driessche. Inertially arbitrary sign patterns with no nilpotent realization. Linear Algebra and its Applications, 421:264-283, 2007.

[13] S.J. Kirkland, J.J. McDonald, and M.J. Tsatsomeros. Sign-patterns which require a positive eigenvalue. Linear and Multilinear Algebra, 41:199-210, 1996.

[14] Q. Lin, D D Olesky, and P. van den Driessche. The distance of potentially stable sign patterns to the unstable matrices. SIAM Journal on Matrix Analysis and Applications, 24:356-367, 2002.

[15] J.J. McDonald, D.D. Olesky, M.J. Tsatsomeros, and P. van den Driessche. On the spectra of striped sign patterns. Linear and Multilinear Algebra, 51:39-48, 2003.

[16] J. Miyamichi. Sign structure of $3 \times 3$ stable matrices and their generalization to higher-order matrices. Electronics and Communications in Japan (Part I: Communications), 71(11):6373, 1988.

[17] R. Pereira. Nilpotent matrices and spectrally arbitrary sign patterns. Electronic Journal of Linear Algebra, 16:232-236, 2007.

[18] H.R. Thieme. Mathematics in Population Biology. Princeton University Press, 2003. 